Walter Fonseca 2,3 Chizuru Misago 4 Paulo Freitas 5 Evanguelia Santos 6 Lucília Fernandes 3 Luciano Correia 2,3

\section{Características sócio-demográficas, reprodutivas e médicas de mulheres admitidas por aborto em hospital da Região Sul do Brasil 1}

\author{
Socio-demographic, reproductive, and clinical \\ characteristics of abortion patients hospitalized \\ in southern Brazil 1
}

1 Projeto financiado pelo Special Programme of Research, Development and Research Training in Human Reproduction, da Organização Mundial da Saúde; pelo Overseas Development Administration, do Reino Unido; e pelo Consel ho Nacional de Desenvolvimento Científico (CNPq).

2 Departamento de Saúde Comunitária, Universidade Federal do Ceará. Rua Professor Costa Mendes 1608, 5o andar, Fortaleza, CE 60431-970, Brasil.

3 Instituto de Pesquisas sobre a Saúde da Mulher, Criança e Adolescente. Rua Silva Jatahy 15 , sala 801 Fortaleza, CE 60165-070, Brasil.

4 Department of Epidemiology, London School of Hygiene and Tropical Medicine Keppel Street, London, WCIE 7HT, UK.

5 Departamento de Medicina Social, Universidade Federal de Santa Catarina.

Campus Universitário Trindade, Florianópolis, SC 88000-000, Brasil.

6 Maternidade Carmela Dutra. Av. Mauro Ramos s/no, Florianópolis, SC 88000-000, Brasil.
Abstract In Brazil, induced abortion is legally al lowed only when necessary to save the woman's life or when pregnancy foll ows rape. Despite this legal constraint, induced abortion is widespread. This study presents findings from 620 cases of incomplete abortion admitted to a major obstetric hospital in Florianópolis, Brazil, between July 1, 1993, and June 30, 1994. Almost one quarter (141) of the abortion cases were induced. About $50 \%$ of the latter patients reported having induced abortion with misoprostol alone or with other methods. Most of the women with induced abortions were young and single (or living without a stable partner), had primary schooling, and were not using any contraceptive method at the time of conception. Among the women who were hospitalized for complications of induced abortion, the clinical characteristics suggest less severe complications than reported in previous studies. Recommendations are made as to the need for confirmatory studies, information regarding cultural perceptions and concepts of abortion, and reasons why low-income women fail to adopt available family planning methods. Key words Epidemiology; Women's Health; Abortion; Reproductive Health; Family Planning

Resumo No Brasil, a indução do aborto élegalmente permitida somente quando necessária para salvar a vida da mul her ou quando a concepção ocorreu de estupro. Apesar das restrições le gais, morais e religiosas, a indução do aborto é amplamente praticada. Com o objetivo de identificar as características do aborto incompl eto nas mulheres admitidas na maternidade pública de Florianópolis, foram entrevistadas 620 mulheres, entre 1o de jul ho de 1993 e 30 de junho de 1994. Com base em dados coletados por mei o de questi onário estruturado, são apresentadas características sócio-demográficas, reprodutivas e médicas dos abortos, classificados como certamente provocados, possivel mente provocados e espontâneos. Entre os 141 casos de aborto provocado, cerca de $50 \%$ das mul heres reportaram uso i solado do Cytotec ${ }^{\circledR}$ (mi soprostol), ou deste associado a outro método abortivo. Os resultados revel am que na população estudada a indução do aborto é prática comum entre mulheres jovens, sol tei ras ou sem parcei ro estável, de bom nível de escolari dade e não usuárias de métodos anti conceptivos. Foi também registrada uma redução do número de complicações graves relacionadas ao aborto provocado admitido ao hospital . Palavras-chave Epidemiologia; Saúde da Mulher; Aborto; Saúde Reprodutiva; Planejamento Familiar 


\section{Introdução}

Os abortos provocados têm sido apontados como uma das principais causas de morbi-mortalidade materna em países onde existem restrições legais a tal prática (The Alan Guttmacher Institute, 1994). A indução do aborto é permitida por lei no Brasil somente quando necessária para salvar a vida da mulher ou quando a concepção ocorreu de um estupro (Código Penal, Artigos 126-129, Decreto-Lei no 2.848 de 7 de dezembro de 1969). Entretanto, sabe-se que a prática do aborto provocado como último recurso para a interrupção de uma gravidez não planejada e não desejada é bastante freqüente entre as mulheres brasileiras, das diferentes classes sociais (Fonseca et al., 1996). Nas grandes metrópoles do País, é comum a prática clandestina do aborto em determinadas clínicas privadas, onde técnicas modernas, como a aspiração uterina a vácuo, são utilizadas. Este procedimento pode custar até US\$1.000,00, restringindo o seu acesso a muIheres de alta renda (Misago, 1994). As mulheres de baixa renda devem recorrer a medidas de alto risco à saúde, como procurar um 'aborteiro' ou se automedicar com drogas abortivas de eficácia não comprovada, geralmente vendidas sem prescrição médica em farmácias (Coelho et al., 1991; Misago, 1994).

É interessante notar que, apesar das restrições legais existentes no Brasil, são raros os casos de prática clandestina de aborto que são levados a julgamento (Osis et al., 1996); tampouco a existência de rígidas normas morais e religiosas consegue impedir que um elevado número de mulheres da população social e economicamente menos favorecida pratique o aborto. Segundo estimativas indiretas, é de aproximadamente 1,4 mil hões o número de abortos provocados ilegalmente a cada ano no País (The Alan Guttmacher Institute, 1994).

A falta de estatísticas oficiais e de estudos com base populacional dificulta uma avaliação da magnitude do problema do aborto no Brasil. Os poucos dados disponíveis sobre os abortos provocados são aqueles obtidos de pesquisas e levantamentos feitos em hospitais (Costa $\&$ Vessey, 1993; Coelho et al., 1993; Fonseca et al., 1996; Fonseca et al., 1997). Sabe-se, entretanto, que complicações por aborto estão entre as principais causas de admissão hospitalar (Schor, 1990; Costa \& Vessey, 1993; Fonseca et al., 1996).

A realização de pesquisas epidemiológicas com pacientes hospitalizadas por complicações relacionadas ao aborto tem especial relevância para o estudo da morbi-mortalidade e estimativas de custos desta patologia. Esta população inclui principalmente casos graves e que requereram hospitalização, estando, portanto, expostos a maiores riscos de seqüel as e óbito. Sabe-se também que o tratamento do aborto no hospital consome parte substancial dos escassos recursos do sistema de saúde (Fonseca et al., 1997).

Nos últimos cinco anos, tem-se observado um aumento substancial do número de casos de aborto admitidos em hospitais da rede pública. Este aumento não é acompanhado por uma elevação proporcional do número total de admissões aos serviços de obstetrícia (Coelho et al., 1993; Fonseca et al., 1996). Esta elevação no número de casos de aborto vem sendo observada desde o fim dos anos 80 e início dos anos 90 (Singh \& Sedgh, 1997). Surpreendentemente, o número de casos de abortos provocados com seqüelas graves e o coeficiente de letal idade têm apresentado acentuada diminuição. É possível que isto esteja relacionado com o crescente uso de misoprostol como método abortivo por mulheres de baixa renda (Coelho et al., 1993; Costa \& Vessey, 1993; Barbosa \& Arilha, 1993; Fonseca et al., 1996; Singh \& Sedgh, 1997). O misoprostol é licenciado no Brasil sob o nome comercial de Cytotec ${ }^{\circledR}$ e tem indicação médica no tratamento de úlceras gástricas e duodenais. A droga tem algum efeito uterotônico, sendo, por isso, contra-indicada para uso durante a gravidez (Downie, 1991). Assim, em razão da disponibilidade do misoprostol como droga abortiva, menor número de mulheres estaria recorrendo a métodos mais invasivos na indução do aborto (Fonseca et al., 1996). Entretanto estudos recentes indicam que a eficácia do misoprostol como abortivo varia entre $40 \%$ e 70\% (Koopersmith \& Mishell, 1996; Bugal ho et al., 1996). Em conseqüência, um percentual elevado de mulheres expostas à droga durante o primeiro trimestre de gestação poderia não abortar, com risco aumentado de malformações congênitas nestes fetos (Fonseca et al., 1993; Gonzales et al., 1993).

O presente estudo buscou investigar características sócio-demográficas, reprodutivas e médicas do aborto nas mulheres admitidas em hospital por complicações associadas à perda da gravidez em Florianópolis, região Sul do país. 


\section{Metodologia}

Florianópolis é uma cidade de porte médio, capital do Estado de Santa Catarina, e tem uma população de aproximadamente 250.000 habitantes. Aproximadamente $90 \%$ dos domicílios dispõem de água tratada e $35 \%$, de rede de esgoto (SESA, 1993). A prestação de serviços em saúde, em sua quase totalidade, é feita de forma gratuita pelos 48 centros de saúde e seis hospitais. Cerca de $80 \%$ das mães fazem prénatal regularmente, e $45 \%$ das mulheres fazem uso adequado de métodos de planejamento familiar. Mais de $98 \%$ dos nascimentos ocorrem em hospital (Sinasc, 1994).

O estudo incluiu 620 mulheres admitidas com diagnóstico de interrupção da gravidez, no serviço de emergência do principal hospital maternidade do município (Maternidade Carmela Dutra), no período compreendido entre 1o de julho de 1993 e 30 de junho de 1994. Antes do início da entrevista, cada paciente foi informada sobre o objetivo do estudo e obtevese seu consentimento para participação, sendo assegurada às participantes a confidencialidade das informações fornecidas. As entrevistas foram conduzidas de forma tão privada quanto possível, em horário conveniente para a muIher durante a sua permanência no hospital. Utilizou-se um questionário padronizado com perguntas sobre condições sócio-econômicase demográficas, história reprodutiva, práticas contraceptivas e abortivas, com uma descrição detal hada sobre os métodos utilizados, quando possível. Foram também revisados os prontuários das internações hospitalares, para a coleta de dados sobre as intercorrências clínicoobstétricas, tempo de permanência no hospital e tratamento recebido. Nenhuma mulher selecionada para a entrevista se recusou a participar do estudo.

Todos os casos de aborto foram reavaliados tendo por base critérios médicos pré-estabelecidos e as respostas colhidas nas entrevistas, sendo classificados em quatro categorias, de acordo com proposta da Organização Mundial da Saúde (WHO, 1987): (a) certamente provocado, quando a mulher admitiu ter provocado o aborto ou quando foram encontrados sinais clínicos de intervenção, tais como laceração cervical e/ ou corpo estranho na vagina ou no útero; (b) provavelmente provocado, quando a mulher não admitiu ter provocado o aborto mas referiu gravidez não planejada e foram encontrados sinais de sepsis ou peritonite; (c) possivelmente provocado, quando somente uma das duas condições listadas anteriormente em (b) esteve presente. Todos outros casos de aborto foram classificados como espontâneos.

O software Epi-Info versão 6.0 (Dean et al., 1994) foi utilizado na preparação e análise dos dados coletados.

\section{Resultados}

Um total de 620 casos de aborto foi investigado durante o período de estudo, e 141 destes $(22,7 \%)$ foram classificados como certamente provocados, 313 (50,5\%), como possivelmente provocados, e 166 (26,8\%), como espontâneos. Não houve registro de casos de aborto provavelmente provocado. Na categoria de abortos certamente provocados, a maioria das mulheres $(94,3 \%)$ admitiu ter tentado terminar a gravidez. Apenas oito $(5,7 \%)$ casos foram classificados como aborto provocado, com base na presença de sinais clínicos de intervenção, tais como laceração cervical e/ ou corpos estranhos na vagina ou no útero.

\section{Características sócio-demográficas}

De acordo com os dados especificados na Tabela 1, observou-se que quanto maior o grupo etário, menor a proporção de mulheres classificadas como tendo certamente provocado o aborto. A proporção de abortos possivelmente provocados seguiu padrão inverso, tendo sido maior, quanto maior o grupo etário. Já a distribuição de abortos espontâneos foi praticamente igual em todos os grupos etários.

Quanto à escolaridade, pôde-se observar que a proporção de mulheres com aborto provocado foi maior, quanto maior o nível de escolaridade. As diferenças encontradas, porém, não foram estatisticamente significativas ( $p=$ 0,47).

Com relação ao estado civil, a proporção de mulheres com aborto certamente provocado foi cerca de quatro vezes maior entre as mulheres solteiras, divorciadas ou viúvas, em relação às mulheres casadas ou em união estável.

Uma alta proporção de mulheres $(86,6 \%)$ era católica, e cerca de um quarto destas foi identificado como tendo provocado o aborto.

A distribuição das mulheres segundo a ocupação e tipo de aborto, com exceção das donas de casa e estudantes, apresentou pouca variação. Entre as donas de casa, a proporção de abortos certamente provocados foi de apenas $15,4 \%$ e entre as estudantes este percentual elevou-se a $48,6 \%(p \varangle 0,05)$. 
Tabela 1

Distribuição de 620 casos de aborto segundo características sócio-demográficas e tipo de aborto. Florianópolis, junho/93 a maio/94.

\begin{tabular}{|c|c|c|c|}
\hline Variáveis & $\begin{array}{l}\text { Certamente } \\
\text { provocado } \\
n=141(\%)\end{array}$ & $\begin{array}{l}\text { Tipo de aborto } \\
\text { Possivelmente } \\
\text { provocado } \\
n=313(\%)\end{array}$ & $\begin{array}{l}\text { Espontâneo } \\
n=166(\%)\end{array}$ \\
\hline \multicolumn{4}{|l|}{ Idade (anos) } \\
\hline$\leq 19$ & $30(29,7)$ & $41(40,6)$ & $30(29,7)$ \\
\hline $20-24$ & $58(31,9)$ & $77(42,3)$ & $47(25,8)$ \\
\hline $25-29$ & $24(17,0)$ & $79(56,0)$ & $38(27,0)$ \\
\hline $30-34$ & $21(18,4)$ & $64(56,1)$ & $29(25,4)$ \\
\hline $35+$ & $8(9,8)$ & $52(63,4)$ & $22(26,8)$ \\
\hline \multicolumn{4}{|l|}{$p<0,001$} \\
\hline \multicolumn{4}{|l|}{ Escolaridade } \\
\hline$\leq 4$ anos & $25(17,6)$ & $75(52,8)$ & $42(29,6)$ \\
\hline $5-8$ anos & $58(23,1)$ & $129(51,4)$ & $64(25,5)$ \\
\hline $9+$ & $58(25,6)$ & $109(48,0)$ & $60(26,4)$ \\
\hline \multicolumn{4}{|l|}{$p=0,47$} \\
\hline \multicolumn{4}{|l|}{ Estado civil } \\
\hline Casada/União estável & $46(11,6)$ & $211(53,3)$ & $139(35,1)$ \\
\hline Solteira/Divorciada/Viúva & $95(42,4)$ & $102(45,5)$ & $27(12,1)$ \\
\hline \multicolumn{4}{|l|}{$p<0,001$} \\
\hline \multicolumn{4}{|l|}{ Religião } \\
\hline Católica & $123(22,9)$ & $260(48,4)$ & $154(28,7)$ \\
\hline Nenhuma & $8(33,3)$ & $12(50,0)$ & $4(16,7)$ \\
\hline Outra & $10(16,9)$ & $41(69,5)$ & $8(13,6)$ \\
\hline \multicolumn{4}{|l|}{$p=0,02$} \\
\hline \multicolumn{4}{|l|}{ O cupação } \\
\hline Dona de casa & $39(15,4)$ & $139(54,9)$ & $75(29,6)$ \\
\hline Prestação de serviços & $35(24,6)$ & $67(47,2)$ & $40(28,2)$ \\
\hline Comércio & $26(25,7)$ & $46(45,5)$ & $29(28,7)$ \\
\hline Estudante & $17(48,6)$ & $14(40,0)$ & $4(11,4)$ \\
\hline Doméstica & $14(25,5)$ & $28(50,9)$ & $13(23,6)$ \\
\hline Desempregada/Dependente & $10(29,4)$ & $19(55,9)$ & $5(14,7)$ \\
\hline$p=0,003$ & & & \\
\hline
\end{tabular}

\section{Características reprodutivas}

A Tabela 2 mostra a distribuição dos casos de aborto segundo características reprodutivas. A menor média de idade gestacional (10,5 semanas) foi observada entre os casos de abortos certamente provocados.

Com relação ao número de filhos vivos, observa-se que aproximadamente um quarto $(26,6 \%)$ das mulheres que referiram não terem filhos vivos foi classificado como tendo tido aborto provocado.

Quanto à ocorrência de aborto prévio, verifica-se que cerca de metade $(48,3 \%)$ das mu-
Iheres com história anterior de aborto provocado foi classificada como tendo provocado o aborto, cerca de $40 \%$, como possivelmente provocado, e apenas $10 \%$ como aborto espontâneo. Entre as mulheres com história anterior de aborto espontâneo, a proporção de casos de aborto provocado foi de apenas $15 \%$.

\section{Características médicas do aborto}

A Tabela 3 apresenta a distribuição de 141 casos de aborto certamente provocado, segundo método anticonceptivo no mês da concepção. A maioria $(53,9 \%)$ das mulheres relatou não fazer uso de qualquer método anticonceptivo e $20,6 \%$ referiu uso de pílula. Um reduzido percentual citou uso de preservativo $(5,7 \%)$.

Os procedimentos usados na indução do aborto das 141 mulheres com aborto provocado são apresentados na Tabela 4. A metade das mulheres (50,4\%) admitiu uso de misoprostol, sozinho ou associado a outros métodos abortivos, para provocar o aborto. A outra metade $(49,6 \%)$ relatou ter usado outros métodos na indução do aborto, sendo mais freqüentemente citados os chás e injeções intramusculares. Foi identificado reduzido número de casos de aborto provocado por inserção de corpos estranhos ou instilação de substância química, por catéter, no colo uterino (5,7\%).

Entre as 71 mulheres que reportaram uso de misoprostol na indução do aborto, as dosagens citadas variaram entre 400 e 5.600 microgramas (Tabela 5). A grande maioria $(62,2 \%)$ referiu ter ingerido a droga e, simultaneamente, tê-la introduzido na cavidade vaginal. Aproximadamente $23,0 \%$ e $15,0 \%$ das mulheres mencionaram o uso exclusivo vaginal ou oral, respectivamente.

A Tabela 6 mostra as características médicas do aborto durante a hospitalização. A maioria das mulheres $(58,6 \%)$ recebeu tratamento e alta médica nas primeiras 24 horas. Entre as mulheres que permaneceram hospitalizadas por mais de três dias, cerca da metade pertencia ao grupo de aborto certamente provocado. Também o tempo médio de hospitalização foi mais elevado para os casos de aborto provocado, com média de 2,1 dias.

Uma alta proporção de mulheres $(88,2 \%)$ não apresentou qualquer sinal clínico de infecção no momento da admissão. Entre os 73 $(11,8 \%)$ casos de aborto com sinais de infecção na admissão, 45,2\% eram certamente provocados. Cerca de um quarto $(25,8 \%)$ das muIheres recebeu tratamento com antibiótico, e a média do número de dias de tratamento com esta medicação $(5,0$; D.P. 2,5$)$ foi mais eleva- 
da nos casos de abortos certamente provocados.

O volume de sangramento após o início do aborto foi relatado como tendo sido superior ao fluxo normalmente observado durante o período menstrual por $16,1 \%$ das mulheres. Nesta categoria, apenas $18 \%$ das mulheres eram casos de aborto certamente provocado. A quase totalidade (96,5\%) das mulheres foi submetida exclusivamente a curetagem para remoção de restos fetais. Não se observaram diferenças estatisticamente significativas entre tipo de aborto e procedimento realizado no tratamento $(p=0,06)$. Foram raros os casos de pacientes submetidas a laparotomia exploradora (1,4\%) e curagem uterina $(1,4 \%)$ por causa de complicações do aborto. Em 10\% dos casos, a expulsão do feto e/ ou restos fetais se processou sem que tivesse sido necessário qualquer procedimento cirúrgico.

\section{Discussão}

Ao analisar os resultados da presente pesquisa, duas limitações devem ser levadas em conta. A primeira refere-se ao tipo de população estudada, que incluiu somente as mulheres admitidas em hospital por complicações relacionadas ao aborto incompleto. Não estão, portanto, representados na amostra estudada os casos de aborto que não buscaram atenção em hospital da rede pública. Assim, os resultados apresentados não devem ser generalizados a outros grupos populacionais. Deve-se também mencionar que a população estudada tem acesso relativamente satisfatório aos serviços de saúde, e alguns achados aqui reportados podem não ser aplicáveis a outras áreas do país. A segunda limitação diz respeito à possível ocorrência de erro sistemático na classificação do aborto. Tentou-se minimizar a ocorrência deste tipo de viés de classificação pela utilização de critérios uniformes para a classificação do aborto. Também foi importante assegurar a boa qualidade das entrevistas, por meio de treinamento cuidadoso das entrevistadoras na coleta de informações.

Por ser o aborto provocado crime passível de punição legal, moral e religiosa, é possível que algumas pacientes que provocaram o aborto tenham preferido declarar ter o mesmo sido espontâneo. Como conseqüência, o número de casos de aborto provocado pode ter sido subestimado. O contrário, por razões óbvias, é pouco provável. Pouco menos de um quarto $(22,7 \%)$ dos casos foi classificado como aborto certamente provocado. Esta estimativa
Tabela 2

Distribuição de 620 casos de aborto segundo características reprodutivas e tipo de aborto. Florianópolis, junho/93 a maio/94.

\begin{tabular}{|c|c|c|c|}
\hline Variáveis & $\begin{array}{l}\text { Certamente } \\
\text { provocado } \\
n=141(\%)\end{array}$ & $\begin{array}{l}\text { Tipo de aborto } \\
\text { Possivelmente } \\
\text { provocado } \\
n=313(\%)\end{array}$ & $\begin{array}{l}\text { Espontâneo } \\
n=166(\%)\end{array}$ \\
\hline \multicolumn{4}{|c|}{ Idade gestacional (semanas) } \\
\hline$\leq 10$ & $80(26,7)$ & $143(47,7)$ & $77(25,7)$ \\
\hline $11-12$ & $24(17,5)$ & $73(53,3)$ & $40(29,2)$ \\
\hline $13-16$ & $28(21,5)$ & $69(53,1)$ & $33(25,4)$ \\
\hline $17+$ & $9(17,0)$ & $28(52,8)$ & $16(30,2)$ \\
\hline \multicolumn{4}{|l|}{$p=0,40$} \\
\hline $\begin{array}{l}\text { Média de idade } \\
\text { gestacional }\end{array}$ & $\begin{array}{r}10,5 \\
(D . P .=3,7)\end{array}$ & $\begin{array}{r}11,6 \\
(D . P .=4,5)\end{array}$ & $\begin{array}{r}11,8 \\
(D . P .=4,5)\end{array}$ \\
\hline \multicolumn{4}{|l|}{ Filhos vivos } \\
\hline Nenhum & $61(26,6)$ & $93(40,6)$ & $75(32,8)$ \\
\hline $1-3$ & $75(21,2)$ & $192(54,2)$ & $87(24,2)$ \\
\hline $4+$ & $5(13,5)$ & $28(75,7)$ & $4(10,8)$ \\
\hline \multicolumn{4}{|l|}{$p<0,001$} \\
\hline \multicolumn{4}{|c|}{ Abortos espontâneos } \\
\hline Nenhum & $121(24,8)$ & $247(50,7)$ & $119(24,4)$ \\
\hline $1+$ & $20(15,0)$ & $66(49,6)$ & $47(35,3)$ \\
\hline \multicolumn{4}{|l|}{$p=0,01$} \\
\hline \multicolumn{4}{|c|}{ Abortos provocados } \\
\hline N enhum & $127(21,5)$ & $301(50,9)$ & $163(27,6)$ \\
\hline $1+$ & $14(48,3)$ & $12(41,4)$ & $3(10,3)$ \\
\hline$p=0,002$ & & & \\
\hline
\end{tabular}

Tabela 3

Distribuição de 141 casos de aborto provocado segundo método anticonceptivo usado no mês da concepção. Florianópolis, junho/93 a maio/94.

\begin{tabular}{lc}
\hline Método anticonceptivo no mês da concepção & Número de casos (\%) \\
\hline Nenhum & $76(53,9)$ \\
Pílula & $29(20,6)$ \\
Ritmo & $13(9,2)$ \\
Coito interrompido & $10(7,1)$ \\
Condom & $8(5,7)$ \\
Outros (DIU, diaframa etc.) & $5(3,5)$ \\
\hline
\end{tabular}




\section{Tabela 4}

Distribuição de 141 casos de aborto provocado segundo método utilizado. Florianópolis, junho/93 a maio/94.

\begin{tabular}{lc}
\hline Métodos & Número de casos (\%) \\
\hline Cytotec $^{\circledR}$ (misoprostol) somente & $39(27,7)$ \\
associado a outros métodos & $32(22,7)$ \\
Outros métodos (chás, outras drogas, injeções etc.) & $70(49,6)$ \\
\hline
\end{tabular}

Tabela 5

Distribuição de 71 casos de aborto provocado segundo dose de misoprostol reportada. Florianópolis, junho/93 a maio/94.

\begin{tabular}{lc}
\hline Dose de misoprostol* (microgramas) & Número de casos (\%) \\
\hline$\leq 400$ & $11(15,5)$ \\
$600-800$ & $13(18,3)$ \\
$1000-1600$ & $27(38,0)$ \\
$1800-2400$ & $11(15,5)$ \\
$2600-5600$ & $9(12,7)$ \\
\hline
\end{tabular}

* 1 comprimido $=200$ microgramas.

é bastante inferior aos valores reportados por estudos recentes, utilizando metodologia semelhante, de três outros estados brasileiros. Em Fortaleza, Nordeste do Brasil, de 4.359 muIheres admitidas ao hospital, em um período de 12 meses, por complicações do aborto, 48\% foram identificadas como tendo provocado o aborto (Fonseca et al., 1996). Um total de 653 mulheres foi admitido por complicações de aborto em Goiás, $40 \%$ das quais foram classificadas na categoria aborto provocado (Viggiano et al., 1996). No Rio de Janeiro, Região Sudeste, foram estudados 1.603 casos de aborto incompleto admitidos no hospital, sendo $50 \%$ classificados como abortos provocados (Costa \& Vessey, 1993). É possível que em Florianópolis, apesar das entrevistas terem sido conduzidas por enfermeiras treinadas e em ambiente privado, tenha havido uma tendência mais acentudada de não informar sobre a indução do aborto. Uma outra explicação seria o maior acesso ao planejamento familiar, prevenindo as gravidezes indesejadas. A realização de estudos complementares que incluíssem informações sobre os conhecimentos relacionados a percepções e conceitos culturais do aborto talvez pudesse esclarecer as diferenças observadas.
Os resultados também indicam que uma parcela importante das mulheres que buscam atenção hospitalar para tratamento de aborto provocado é constituída por jovens, solteiras, com bom nível de escolaridade, sem ocupação remunerada, e não usuárias de métodos modernos de anticoncepção. Enquanto estes resultados confirmam os achados de pesquisas realizadas no Nordeste, Centro e Sudeste do País (Fonseca et al., 1996; Viggiano et al., 1996; Costa \& Vessey, 1993), eles são marcadamente diversos de achados de estudos em outros países da América Latina (Fortney, 1981).

A média da idade gestacional não ultrapassou 12 semanas, e nos casos de aborto certamente provocado foi de 10,5 semanas. Este valor é semelhante ao reportado em Fortaleza (11,4 semanas) (Fonseca et al., 1996) e inferior ao referido em outros estudos de vários países latino-americanos, na década passada (Fortney, 1981). Nos grupos de aborto certamente provocado e possivelmente provocado, uma proporção elevada de mulheres reportaram ter três ou menos filhos vivos. Aproximadamente um terço destas mulheres relatou não ter nenhum filho vivo, indicando a pouca experiência com eventos relacionados à maternidade. Entre os 141 casos de aborto provocado, foi alta a proporção (54\%) de mulheres que não utilizava qualquer método contraceptivo no mês da concepção. Somente 5,7\% das mulheres neste grupo informou o uso de preservativos. Entre as mulheres sem parceiros estáveis, as razões mais freqüentemente citadas para o não-uso de algum método anticonceptivo foram o "descuido", "não esperava ter rel ação sexual" e "medo de efeitos adversos". A indisponibilidade de métodos contraceptivos foi mencionada por menos de $5 \%$ das mulheres. Estes resultados coincidem com os achados do estudo realizado em Fortaleza (Fonseca et al., 1996) e indicam que também em Florianópolis deveria ser promovido o uso de preservativos na prevenção da gravidez não planejada e das doenças sexualmente transmissíveis, incluindo a Sida/Aids. Também deveriam ser testadas intervenções que pudessem aumentar a utilização de métodos contraceptivos modernos, incluindo a anticoncepção de emergência.

Mais da metade $(50,4 \%)$ das mulheres com aborto provocado relataram o uso de misoprostol, isolado ou em combinação com outros métodos, na indução do aborto. Freqüências também elevadas foram reportadas em estudos em Fortaleza (66,0\%), Rio de Janeiro $(57,0 \%)$ e Goiânia (39,0\%), indicando que este método de aborto é prática comum entre mu- 
Iheres que buscam atendimento em hospitais da rede pública (Fonseca et al., 1996; Viggiano et al., 1996; Costa \& Vessey, 1993).

Os dados mostram que aproximadamentea metade $(45,2 \%)$ dos casos de aborto provocado apresentou sinais clínicos de infecção à admissão e foi tratado com antibióticos $(47,5 \%)$, durante uma média de cinco dias. Cerca de um quinto (18\%) das mulheres com aborto provocado apresentou hemorragia grave à admissão. Estes dados indicam que os casos de aborto provocado admitidos em hospital em Florianópolis apresentaram um grau de gravidade superior ao descrito em estudos recentes em Fortaleza e em Goiânia (Fonseca et al., 1996; Viggiano et al., 1996), mas ainda assim bastante inferior ao descrito por estudos em outros países latino-americanos (Fortney, 1981; Bailey et al., 1988). É possível que em Florianópolis as mulheres que provocaram o aborto tenham buscado atendimento em serviços de saúde mais tardiamente, aumentando, dessa forma, os riscos de hemorragia e infecção grave. Estudos epidemiológicos complementares são necessários para esclarecer as diferenças observadas.

É notável o achado de que praticamente todas as mulheres (98\%) foram submetidas a dilatação e curetagem ( $D \& C$ ) para a remoção de restos fetais. Este método é utilizado no tratamento do aborto na grande maioria dos hospitais da rede pública do Brasil. Estudos de diversos países desenvolvidos reportam ser a aspiração intra-uterina a vácuo mais eficaz, mais segura e de menor custo se comparada à $D \& C$ no tratamento do aborto incompleto (Tietze $\&$ Lewit, 1972; Johnson et al., 1993). Nestes países, assim como na maioria dos serviços privados do Brasil, utiliza-se a técnica de aspiração a vacuo há mais de uma década. Estudo recente em hospital da região Nordeste do País documentou a notável redução de cerca de $50 \%$ no custo médio do tratamento e de aproximadamente $80 \%$ na duração da estadia hospitalar, nos casos de pacientes com aborto incompleto tratadas com aspiração manual a vácuo (AMV), em comparação com mulheres tratadas com D\&C. Além disso, dados preliminares de pesquisa utilizando metodologia qual itativa revelam a boa aceitação do AMV, tanto por profissionais de saúde, como por mulheres tratadas com este método (Fonseca et al., 1997).

Em resumo, o presente estudo apresenta vantagens e limitações próprias de estudos descritivos do tipo transversal. O recrutamento de mulheres em hospital permitiu, de forma relativamente barata e simples, identificar
Tabela 6

Características médicas de 620 abortos admitidos em hospital segundo tipo de aborto. Florianópolis, junho/93 a maio/94.

\begin{tabular}{|c|c|c|c|}
\hline Variáveis & $\begin{array}{l}\text { Certamente } \\
\text { provocado } \\
n=141(\%)\end{array}$ & $\begin{array}{l}\text { Tipo de aborto } \\
\text { Possivelmente } \\
\text { provocado } \\
n=313(\%)\end{array}$ & $\begin{array}{l}\text { Espontâneo } \\
n=166(\%)\end{array}$ \\
\hline \multicolumn{4}{|l|}{ Dias de hospitalização } \\
\hline$\leq 1$ & $70(19,3)$ & $187(51,5)$ & $106(29,2)$ \\
\hline $2-3$ & $47(22,7)$ & $104(50,2)$ & $56(27,1)$ \\
\hline $4+$ & $24(48,0)$ & $22(44,0)$ & $4(8,0)$ \\
\hline \multicolumn{4}{|l|}{$p<0,001$} \\
\hline $\begin{array}{l}\text { Média de dias de } \\
\text { hospitalização }\end{array}$ & (D.P. $=\begin{array}{r}2,1 \\
1,4)\end{array}$ & $\begin{array}{r}1,7 \\
(D . P .=1,2)\end{array}$ & (D.P. $=\begin{array}{r}1,5 \\
0,9)\end{array}$ \\
\hline \multicolumn{4}{|l|}{ Sinais de infecção à admissão } \\
\hline Sim & $33(45,2)$ & $27(37,0)$ & $13(17,8)$ \\
\hline Não & $108(19,7)$ & $286(52,3)$ & $153(28,0)$ \\
\hline \multicolumn{4}{|l|}{$p<0,001$} \\
\hline \multicolumn{4}{|l|}{ Tratamento com antibióticos } \\
\hline Sim & $76(47,5)$ & $68(42,5)$ & $16(10,0)$ \\
\hline Não & $65(14,1)$ & $245(53,3)$ & $150(32,6)$ \\
\hline \multicolumn{4}{|l|}{$p<0,001$} \\
\hline $\begin{array}{l}\text { Média de dias de tratamento } \\
\text { com antibiótico }\end{array}$ & $\begin{array}{r}5,0 \\
(D . P .=2,5)\end{array}$ & $\begin{array}{r}4,2 \\
(D . P .=2,5)\end{array}$ & $\begin{array}{r}4,2 \\
(D . P .=3,4)\end{array}$ \\
\hline \multicolumn{4}{|l|}{ Hemorragia grave à admissão } \\
\hline Sim & $18(18,0)$ & $44(44,0)$ & $38(38,0)$ \\
\hline Não & $123(23,7)$ & $269(51,7)$ & $128(24,6)$ \\
\hline \multicolumn{4}{|l|}{$p=0,02$} \\
\hline \multicolumn{4}{|l|}{ Procedimentos realizados } \\
\hline Curetagem & $138(23,1)$ & $303(50,7)$ & $157(26,3)$ \\
\hline Nenhum & 0 & $9(56,3)$ & $7(43,7)$ \\
\hline Outros & $3(50,0)$ & $1(16,7)$ & $2(33,3)$ \\
\hline
\end{tabular}

um número suficiente de casos de aborto para a investigação de seus determinantes. As informações coletadas permitiram inúmeras análises, desde questões mais abrangentes, como o perfil sócio-demográfico, reprodutivo e de morbidade, até questões mais singulares, como método utilizado na indução do aborto e a identificação das principais razões para não-uso de métodos modernos de anticoncepção.

Resultados deste estudo confirmam mais uma vez a necessidade de identificação de novas estratégias de planejamento familiar e rotinas hospitalares que contribuam para a meIhoria do tratamento do aborto. 


\section{Agradecimentos}

Os autores agradecem aos funcionários da Maternidade Carmela Dutra pela colaboração prestada durante a realização do estudo.

\section{Referências}

BAILEY, P. E.; SAAVEDRA, L. L.; KUSBNER, L.; WELSH, M. \& JANOWITZ, B., 1988. A hospital study of illegal abortion in Bolívia. PAHO Bulletin, 22:27-41.

BARBOSA, R. M. \& ARILHA, M., 1993. The Brazilian experience with Cytotec. Studies in Family Planning, 24:236-240.

BUGALHO, A.; FAUNDES, A.; JAMISSE, L.; USFÁ, M .; MARIA, E. \& BIQUE, C., 1996. Evaluation of the effectiveness of vaginal misoprostol to induce first trimester abortion. Contraception, 53:243246.

COELHO, H. L. L.; MISAGO, C.; FONSECA, W.; SOUSA, D. S. C. \& ARAUJO, J. M. L., 1991. Selling abortifacients over the counter in pharmacies in Fortaleza, Brazil. The Lancet, 338:247.

COELHO, H. L. L.; TEIXEIRA, A. C.; SANTOS, A. P.; FORTE, E. B.; MORAES, S. M.; LA VECHIA, C.; TOGNONI, G. \& HERXHEIMER, A., 1993. Misoprostol and illegal abortion in Fortaleza, Brazil. Lancet, 341:1.261-1.263.

COSTA, S. \& WESSEY, M. P., 1993. Misoprostol and illegal abortion in Rio de Janeiro, Brazil. Lancet, 341:1.258-1.261.

DEAN, A. G.; DEAN, J. A.; COULOMBIER, D.; BRENDEL, K. A.; SMITH, D. C.; BURTON, A. H.; DICKER, R. C.; SULLIVEN, K.; FAGAN, R. F. \& ARNER, T. G., 1994. Epi Info, Version 6: A Word Processing Database, and Statistics Program for Epidemiology on Microcomputers. Atlanta: Center for Disease Control and Prevention.

DOWNIE, W. W., 1991. Misuse of misoprostol. Lancet, 338:247.

FONSECA, W.; ALENCAR, A. J. C.; PEREIRA, R. M. M.; MISAGO, C., 1993. Congenital malformation of the scalp and cranium after failed first trimester abortion attempt with misoprostol. Clinical Dysmorphology, 2:76-80.

FONSECA, W.; MISAGO, C.; CORREIA, L.; PARENTE, J. A. M. \& OLIVEIRA, F. C., 1996. Determinantes do aborto provocado entre mulheres admitidas em hospitais em localidade da região Nordeste do Brasil. Revista de Saúde Pública, 30:13-18.

FONSECA, W.; MISAGO, C.; FERNANDES, L.; CORREIA, L. \& SILVEIRA, D., 1997. Uso de aspiração manual a vácuo na redução do custo e duração de internamentos por aborto incompleto em Fortaleza, Brasil. Revista deSaúdePública, 31:1-5.

FORTNEY, J. A., 1981. The hospital resources to treat incomplete abortions: exemples from Latin America. Public Health Reports, 96:574-579.

GONZALEZ, C. H.; VARGAS, F. R.; PERES, A. B. A; KIM, C. A.; BRUNORI, D.; MARQUES-DIAS, M. J.; LEONE, C. R.; CORREA NETO, J.; LLERENA JR., J.
C. \& ALMEIDA, J. C. C., 1993. Limb deficiency with or without Möbious sequence in seven Brazilian children associated with misoprostol use in the first trimester of pregnancy. American Journal of Medical Genetics, 47:59-64.

JOHNSON, B. R.; BENSON, J.; BRADELY, J. \& ORDOÑEZ, R., 1993. Cost and resource utilization for the treatment of incomplete abortion in Keny a and Mexico. Social Sciences and Medicine, 36:1.443-1.453.

KOOPERSMITH, T. B. \& MISHELL JR., D. R., 1996. The use of misoprostol for termination of early pregnancy. Contraception, 53:237-242.

MISAGO, C., 1994. Preventing unsafe abortion and limiting its consequences: what can be done? Canguru, 2:172-177.

OSIS, M. J. D.; HARDY, E.; FAUNDES, A. \& RODRIGUES, T., 1996. Dificuldades para obter informações da população de mulheres sobre aborto ilegal. Revista de Saúde Pública, 30:444-451.

SCHOR, N., 1990. Investigação sobre a ocorrência de aborto em pacientes de hospital de um centro urbano do Estado de São Paulo, Brasil. Revista de Saúde Pública, 24:144-151.

SESA (Secretaria da Saúde), 1993. Indicadores de Saúde. Florianópolis: Secretaria da Saúde do Município de Florianópolis, Santa Catarina.

SINASC (Serviço de Informação sobre Nascidos Vivos), 1994. Registro dos nascidos vivos. Prefeitura Municipal de Florianópolis, Santa Catarina.

SING, S. \& SEDGH, G., 1997. The relationship of abortion to trends in contraception and fertility in Brazil, Colombia and Mexico. International Family Planning Perspectives, 23:4-14.

THE ALAN GUTTM ACHER INSTITUTE, 1994. Aborto Clandestino: Uma Realidade Latino-Americana. Nova York: AGI.

TIETZE, C. \& LEWIT, S., 1972. Joint program for the study of abortion (JPSA): early medical complications of legal abortion. Studies in Family Planning, 3:97-119.

WHO (World Health Organization), 1987. Protocol for Hospital Based Descriptive Studies of Mortality, Morbidity Related to Induced Abortion. Geneva: WHO Task Force on Safety and Efficacy of Fertility Regulating Methods.

VIGGIANO, M. G. C; FAÚNDES, A.; BORGES, A. L.; VIGGIANO, A. B. F.; DE SOUZA, G. R. \& REBELLO, I., 1996. Disponibilidade de misoprostol e complicações de aborto provocado em Goiânia. Jornal Brasileiro deGinecologia, 106:55-61. 